\title{
Germanica
}

\section{Marlen Haushofer : écrire pour transcender sa condition de femme}

Marlen Haushofers Schreiben als Überwindungsversuch ihrer Frauenbedingung. Marlen Haushofer : Writing to transcend one's condition as woman.

\section{Régine Battiston}

\section{OpenEdition}

Journals

Édition électronique

URL : http://journals.openedition.org/germanica/1050

DOI : 10.4000/germanica. 1050

ISSN : 2107-0784

Éditeur

Université de Lille

Édition imprimée

Date de publication : 30 juin 2010

Pagination : 61-72

ISBN : 9782913857254

ISSN : 0984-2632

\section{Référence électronique}

Régine Battiston, «Marlen Haushofer : écrire pour transcender sa condition de femme », Germanica

[En ligne], 46 | 2010, mis en ligne le 01 juin 2012, consulté le 06 octobre 2020. URL : http://

journals.openedition.org/germanica/1050 ; DOI : https://doi.org/10.4000/germanica.1050

Ce document a été généré automatiquement le 6 octobre 2020.

(c) Tous droits réservés 


\title{
Marlen Haushofer : écrire pour transcender sa condition de femme
}

\author{
Marlen Haushofers Schreiben als Überwindungsversuch ihrer Frauenbedingung. \\ Marlen Haushofer : Writing to transcend one's condition as woman.
}

Régine Battiston

L'œuvre de l'écrivain autrichien Marlen Haushofer (1920-1970) ${ }^{1}$ illustre la difficulté d'être une femme et de vivre physiquement et psychologiquement dans cette différence, dans la société autrichienne du milieu du xxe siècle. Il s'agit de la difficulté d'avoir une parole propre et la maîtrise de ses actes, la possibilité de se prendre en charge financièrement et d'assumer ces découvertes par le biais de l'écriture, tout en constatant les freins à cette émancipation et les limites imposées par la société. Les écrits de Haushofer montrent la capacité des femmes à créer des œuvres littéraires, qui, en dehors du témoignage d'un point de vue et d'une époque, parlent à des lecteurs du xxie siècle de problèmes récurrents qui transcendent les époques et les genres littéraires. C'est une écriture de la différence avec un autre regard, celui de la perspective de la femme, de la vue d'une femme qui a eu de grandes difficultés dans son émancipation hors de la tutelle masculine, hors du jeu des valeurs de la société de son époque, sclérosée par des règles patriarcales d'un temps pas encore révolu. Elle montre par des témoignages d'essais de vies de couples, où aucun des scénarios proposés ne dure dans le temps, la solitude de la femme, rejetée hors du champ des valeurs d'une société phallocratique. La femme de Haushofer est tenue à l'écart, lorsqu'elle dérange par son questionnement et son mode de vie. Sa littérature présente une société crispée sur ses valeurs ancestrales, évitant les questions existentielles et le droit au bonheur individuel, faite de personnages évoluant dans des instants de vie privée. La remise en question du principe de réalité de la vie est un sujet majeur dans son œuvre. C'est un combat engagé contre les habitudes de pensée. Il repose sur le rapport de l'œuvre à la temporalité et à l'espace, la notion de limite et ses modes de transgression, le rapport entre l'inconscient narratif et l'émancipation de la femme. Si son œuvre s'est vue décerner quelques prix littéraires du vivant de l'auteur, elle a eu peu de succès auprès 
des lecteurs et a été redécouverte dans les années 1980 par les féministes (américaines avant tout), pour être mieux étudiée et reconnue depuis lors.

\section{L'expression d'une douleur existentielle ou vivre dans un monde fait par et pour les hommes}

2 Marlen Haushofer a tenté de montrer dans son œuvre les frustrations et les aspects refoulés du quotidien ou de la vie de femmes bourgeoises des années 1950-1970. Elle a mis en lumière les comportements féminins induits, en présentant leur banalité et leur présence quotidienne à travers ses héroïnes. Ses récits dévoilent autant la culpabilité féminine que la victimisation des femmes par leur compagnon, la société qui les entoure, que par elles-mêmes. Certaines de ces femmes sont des écrivains, ce statut n'allant pas de soi dans une société basée sur le principe d'une femme d'intérieur dévouée à son foyer et à sa famille. Pour Haushofer, il s'agit d'avoir, comme le demandait Virginia Woolf en 1929, Une chambre à soi, un lieu où se retrouver seule chez soi, ce thème qui lui était personnel est devenu récurrent dans toute l'œuvre. Celle-ci dévoile un monde tourmenté et déchiré par les conflits entre les êtres, et le sentiment amoureux en est le ressort principal. Ses héroïnes sont des femmes écartelées entre leur situation sociale et privée : ainsi, tout en participant au fonctionnement de la société, les femmes se trouvent reléguées, de par leur sexe et la tradition occidentale, dans une position d'infériorité systématique par rapport aux hommes (notamment de par leur métier et leurs revenus) et sont exclues du langage et du pouvoir. Toutes les héroïnes voient leur relation de couple échouer, elles tentent de sauver les apparences face à une société catholique et bien-pensante, mais le naufrage psychologique les guette. Leur impossibilité à résoudre un conflit d'Électre les tient liées à leur enfance, paradis perdu dont elles ont été définitivement chassées. Elles évoluent sous le signe de l'enfermement et de la claustration, leur vie sociale hors de leur foyer est ténue et chaque relation avec l'Autre masculin est marquée du sceau de l'échec. Elles se replient sur elles-mêmes et logiquement le thème de la solitude traverse toute l'œuvre, c'est une solitude de la femme face à autrui avant tout. Le mal être est le lot commun de tous les récits. La maladie personnelle qui apparait rapidement est prétexte d'introspection et toutes les héroïnes souffrent de claustration et de troubles du comportement, sont minées par une profonde angoisse existentielle. La mort sous-jacente accompagne le mal-être individuel, comme une forme de précipice constamment présent.

Dans le roman La Porte dérobée (Die Tapetentür, 1957)² on trouve des réflexions sur les sentiments et les relations impossibles entre les sexes, notamment à travers des exercices d'introspection de la part de l'héroïne Annette, qui utilise la technique du journal comme thérapie. L'action est rendue dans une partie du roman à travers ce prisme (récit à la première personne). L'héroïne y consigne ses réflexions sur la vie et les gens autour d'elle et pratique l'auto-analyse en faisant de l'introspection. Les propos qu'elle y note à son égard, frisent le dénigrement, le reniement et le renoncement de soi. Le journal fait fonction d'exutoire et de thérapie, il est un moyen de sonder son moi, de se mettre à nu. Comme nous l'avons noté dans une étude antérieure, « le livre transcrit une philosophie existentielle pessimiste. Parfois elle est prise d'accès de panique dans la rue, d'une peur d'être seule dans un monde humain hostile. Personnage sensible et facilement blessable, le quotidien la fatigue et elle succombe vite à des troubles psychiques $[. ..] »^{3}$. Il faut noter son attitude fataliste, sa solitude et 
l'absence d'enfants, comme dans tous les romans. La maternité n'a pas sa place dans le conflit entre les sexes, alors que c'est une des fonctions sociales qui est dévolue à la femme dans la société autrichienne de cette époque. Dans la claustration où la tient la dépression nerveuse qui la saisit, elle fuit dans le fantasme. Le passage crucial du roman, celui du rêve de la porte dérobée, relève de la symbolique de la porte et du passage, accès vers la vie, à travers l'accouchement qui se prépare en elle, mais aussi vers la mort, celle de l'enfant qu'elle porte et il annonce aussi la fin de sa relation amoureuse avec son compagnon. L'image de la femme est présentée comme une galerie de portraits dans une liste de conquêtes du Don Juan qu'est son compagnon. Elle est complétée par la fausse image de l'épouse, femme au foyer parfaite qui ne s'épanouit pas (traits autobiographiques de l'auteur). Ici comme dans les autres romans, les relations ambivalentes avec les hommes provoquent des troubles de l'identité.

L'auteur opère une remise en question de l'ordre moral, de l'éducation et de la religion dans son pays. Dans toute l'œuvre la notion de limite est présente et joue un rôle de premier plan. Dans le roman Die Tapetentür qui est en partie un récit d'inspiration autobiographique, le personnage d'Annette a des difficultés avec son Moi. Elle est enceinte, lorsqu'elle remarque qu'elle n'aime pas l'homme avec lequel elle vit. Cette prise de conscience la précipite dans une crise dépressive. Au centre du récit se trouve un rêve qui ressemble à un fantasme, celui de la Porte dérobée ("die Tapetentür »), d'où le titre du roman. Il contient les symboles de son enfance, de l'absence et de l'abandon de son père, d'un traumatisme très mal vécu. Intéressant est le cryptage de ce rêve très fourni en signes symboliques, qui constitue la clé du problème existentiel d'Annette. Le retour par le passage de la porte dérobée (comme un rite de passage) vers son enfance est aussi un retour vers un état intra-utérin. La configuration du terrain et son décor le prouvent. Il s'agit d'une pulsion de régression, motivée par un état dépressif extrême, de l'accession à un refuge, ceci au moment où l'accouchement de l'enfant qu'elle porte se prépare. Le franchissement de cette limite montre en condensé les symboles de son enfance, le décor de la maison familiale, la rivière, le chien jaune ${ }^{4}$. Ce « parcours initiatique » par la transgression de la limite de la porte dérobée, l'amène à une prise de conscience, celle de l'absence du père dans le passé, du compagnon dans son présent. Elle a également accès au monde chiffré des significations psychiques codées. L'approche psychanalytique du roman et l'analyse de la symbolique du passage de la porte montrent la distance des personnages masculins, évoluant dans un monde fermé aux héroïnes, reléguées par l'auteur dans un univers parallèle. L'étude de la relation du couple montre des aspects très différents, vus par le regard d'une femme, notamment l'exclusion des femmes du monde des hommes, leur difficulté d'expression (quel langage pour dire quoi ?), leur rôle et leur place dans la société des hommes, en définitive. Le décalage du langage entre les deux sexes constitue à lui seul une limite infranchissable. Dans le roman Le Mur invisible (Die Wand, 1963) ${ }^{5}$, Haushofer érige physiquement un mur transparent que son héroïne sans nom ne peut traverser. Celle-ci se retrouve ainsi isolée dans un décor alpestre et idyllique qui la tient prisonnière. Contrairement au roman précédent, cette limite ne peut pas être franchie, même si la vision qui tient lieu de scénario à ce roman peut être considérée également comme un fantasme. La symbolique et la fonction de ce mur invisible, limite lisse et transparente, insidieuse aussi, sont parlantes. Il sépare deux mondes pour lesquels il est difficile d'opter librement: d'un côté la claustration et la solitude dans une semi-idylle qui ne parvient pas à endiguer la violence, de l'autre côté la mort (tous les personnages et les animaux sont figés au-delà de ce mur, tout comme ils le sont d'ailleurs dans la vision de 
la porte dérobée). Ici aussi la claustration accompagne nécessairement l'enfermement dans un monde étrange de solitude, tout comme cela est le cas dans le roman Dans la Mansarde (Die Mansarde, 1969) ${ }^{6}$. Dans tous ces romans, la situation de l'héroïne entre liberté, enfermement et solitude est fascinante. La vision d'apocalypse dans Die Wand peut être mise en parallèle avec celle du retour et de la régression dans Die Tapetentür, les deux romans constituent des transgressions de limites : dans le second il s'agit d'un impossible retour vers le passé révolu de l'enfance, vers un monde d'hier meilleur, dans le premier c'est un enfermement hors du monde humain, où tout est mort, dans un «no man's land» en apparence idyllique, fait de solitude et de retour à une vie primitive au sein de la nature (qui rappelle un peu le mythe du bon sauvage, dans une version post-industrielle). Les deux situations mettent en lumière le «non-lieu » dévolu à la femme dans la société et la polarité des sexes ne trouve de solution que dans la violence et le conflit.

\section{L'Autre masculin}

5 À travers les destins de ces hérö̈nes minées constamment par une douleur existentielle, dont l'origine multifactorielle est difficile à définir, le rapport à l'altérité devient difficile, voire impossible. La difficulté des relations avec le Masculin, qu'il s'agisse du couple ou de la figure du père s'ajoute alors à leur douleur de vivre. Cette difficulté est d'ailleurs, depuis la sortie de l'enfance, un des facteurs de déstabilisation du Moi féminin. Une des questions centrales de l'œuvre de Haushofer est la quête de l'identité féminine par l'écriture, question qui se pose à travers les destins des héroïnes, leurs échecs de vie commune et leur difficulté à se trouver une vie supportable dans la société. Leurs destins montrent une difficulté à communiquer et à vivre avec autrui, ce qui reflète une manière d'être au monde et de se situer par rapport à son entourage. La scission du Moi en est le résultat. Dans le rêve-fantasme qui se trouve au centre du roman Die Tapetentür trône la figure mythique du " père absent-amant-Don Juan » tout à la fois. La vision se termine par l'exclusion du féminin. Le rapport entre la claustration, l'absence du père, ainsi que le rejet de la fille par lui, devient évident (même sans l'aide de la psychanalyse) ${ }^{7}$. L'analyse de l'instance paternelle et la constatation de la simple évocation de la mère souligne une absence du père doublée d'une vacuité du rôle de la mère, quand l'enfant n'est présenté qu'à travers des images de mort et de suicide de la mère. Nous soulignons que :

\footnotetext{
Marlen Haushofer a su mettre en évidence le principe de fonctionnement d'une instance paternelle symbolique qui se base sur l'absence du père réel. Par la première absence du père (départ aux USA) l'enfant se retrouve dans une attitude de stérilité psychologique et de stagnation psycho sexuelle. Le deuxième départ définitif $d u$ père démarre un processus de substitution - images du passé, souvenirs, dans lequel Gregor fonctionne comme Ersatz - ce processus aboutit à l'absence finale du conjoint. L'enfant mort-né fait aussi partie de cette chaîne de substitution. La mort de l'enfant (qui ne joue pas son rôle de substitut affectif pour Annette) et la séparation avec Gregor signifient la fin de la stagnation mélancolique et le début d'un vrai travail de deuil. À la fin on a l'impression qu'elle a surmonté la fixation au père, elle détruit en tout cas volontairement son journal, signe qu'elle n'en a plus besoin ${ }^{8}$.
}

La manière de vivre schizophrénique des héroïnes semble être pour Haushofer une condition ou une conséquence du travail d'écriture. Toutes les femmes sont exclues du territoire masculin, soumises à l'autorité du père et à son absence, elles sont 
cantonnées (ou se cantonnent elles-mêmes faute d'avoir l'imagination et le courage de transcender cet interdit virtuel) dans le quotidien, qui devient le territoire de la femme par excellence. On mesure alors la difficulté du combat contre les habitudes de pensée, le poids du regard de la société sur des femmes isolées dans de petites villes de province, la violence entre les sexes et le silence - le tout étant basé sur l'ambigüité de la propre vie privée de l'auteur à la recherche d'alternatives de vie.

Cette littérature féminine n'est pas, on le constate aisément, une littérature du plaisir et de la joie de vivre, les problèmes qu'elle soulève sont des témoignages d'un malaise existentiel individuel profond. Il est partagé par beaucoup de femmes-écrivains dont l'œuvre et la vie ont été marquées par la Deuxième Guerre mondiale. De ce fait on retrouve tous ces thèmes de façon nuancée et différemment illustrés dans la littérature autrichienne, dans laquelle le malaise existentiel privé prend une dimension de «malaise de civilisation ».

\section{Le rapport au paysage}

8 Le choix du paysage n'est jamais anodin pour un écrivain, si pour Marlen Haushofer la petite ville où elle a vécu et les montagnes de son enfance sont des décors incontournables, elle parle toujours de choses qu'elle connaît et qu'elle maîtrise bien. Elle utilise surtout les paysages de sa région natale, la Haute-Autriche, en les recomposant dans ses romans. Ils offrent des décors en partie campagnards, même si ses héroïnes vivent en ville, la campagne les attire toujours. Les descriptions sont inspirées de paysages réels qui sont recomposés dans ses récits. Elle a apporté des contributions au roman de type alpestre. Le roman Die Wand est à mi-chemin entre un roman fantastique et une vision. Les paysages utilisés et leur composition, la fonction de cette nature-mère et surpuissante, la place de la femme dans sa solitude, livrée à son destin, sont les grands thèmes principaux. Certains passages sont presque des contemplations mystiques. On trouve ici des aspects panthéistes et la symbolique de la forêt est accentuée. Cet élément revient dans toutes ses œuvres et prend place dans sa réflexion comme élément féminin de l'inconscient et des pulsions difficiles à prévoir et à contrôler. La proximité des animaux et notamment la présence fidèle et bienveillante de la figure du chien rappelle l'enfance de l'auteur. Si ce roman est du domaine de la vision, Die Tapetentür reste dans celui du fantasme, les deux se rejoignant dans une représentation fantastique et symbolique d'inspiration autobiographique. Marlen Haushofer montre dans ce dernier roman une courte description d'un paysage connu, celui de sa maison natale et de ses environs, intégrée dans le récit en tant qu'élément fantastique. Cette description intervient comme composante d'un rêve-fantasme, avant l'accouchement prématuré de la narratrice qui va perdre l'enfant qu'elle porte. Le chien, qui reste le personnage tutélaire de cette excursion rêvée, constitue un élément fondamental dans la galerie des portraits de l'œuvre de l'auteur (le rapport très fort avec les animaux y remplace celui avec les humains).

9 Marlen Haushofer reste centrée sur les paysages autrichiens qu'elle a connus et qu'elle utilise en les recomposant dans des récits mi-fantastiques. Elle ne fait pourtant pas partie de la veine de l'Alpenroman, mais plutôt des romans psychologiques féminins des années 1950 et l'écriture des paysages constitue pour elle plus qu'un choix de décor rassurant ou déstabilisant, car il véhicule des notions d'humanisme face à un monde hostile et étriqué, dans une mise en question du principe de réalité de la vie. Dans le 
roman Die Wand, la tentation du retrait hors de la société, est grande, l'aventure ressemble à une robinsonnade, forme de régression vers une vie primitive, au sein de la nature hostile, vie difficile pour une femme seule livrée à elle-même, exposée aux éléments naturels et aux saisons. La nature n'y est pas présentée comme idyllique, là aussi la violence entre les sexes est présente, dans un retournement nécessaire à la survie: l'héroïne tue le chasseur, car il a tué son chien. Ce roman ressemble à la récapitulation de l'histoire de l'humanité perpétrée ici par une femme, on y retrouve le thème de la solitude dans un paysage apocalyptique, car malgré le mur, la nature reprend ses droits. Le personnage féminin doit se trouver seul une survie, sans alter ego, et surtout sans compagnon humain. La situation qui fait penser à un lendemain de catastrophe ou de cataclysme mondial de type apocalypse, souligne la proximité avec le monde animal, le rapport homme-chien et chat, ici aussi la symbolique animale montre la profonde humanité et tolérance de l'œuvre de Haushofer.

Si la femme refuse la communication, elle est consciente du rôle subalterne dans lequel elle est emprisonnée. Le mur lui permet, certes dans la douleur, de se réaliser, il constitue aussi une remise en question du principe de réalité de la vie, d'un monde hostile et étriqué.

11 Marlen Haushofer utilise beaucoup de lieux imaginaires, où les personnages féminins semblent préservés, pour être, de façon paradoxale, davantage exposés à la violence des relations interpersonnelles. Elle parvient à établir un contraste entre le cadre calme et idyllique et les drames qui se jouent entre les personnages et aussi à l'intérieur d'eux. Le contact direct avec la nature et surtout les animaux, apparaît comme rassurant et apaisant. Une critique de la civilisation est sous-jacente à tous ses récits, ainsi qu'une interrogation sur la fin de l'humanité. L'œuvre fut aussi un travail d'anthropologie pour l'auteur. Elle met en opposition le chaos de la civilisation, malgré son confort matériel, et l'innocence d'une vie proche de la nature. Cette dichotomie reflète le fossé entre les sexes, qui est très bien rendu dans son œuvre. Elle suggère un profond malaise, qui est une critique d'un ordre patriarcal (une vue que l'auteur partage avec toutes les féministes et surtout avec Ingeborg Bachmann). Si Haushofer opte pour une démonstration par l'utopie (ses romans se déroulent en partie dans un monde irréel), par des visions d'apocalypse, en faisant appel à la subjectivité, son œuvre véhicule pourtant un message d'anti-utopie. Elle parvient à mettre en lumière, de façon magistrale, la situation d'aliénation dans laquelle se trouve la femme.

Les paysages sont une problématique des plus intéressantes dans son œuvre. Il faut noter que certains titres de ses œuvres sont des métaphores topographiques désignant des lieux clos à l'intérieur d'une maison. À la lecture de ses romans, il apparaît un complexe de claustration qui parcourt toute l'œuvre et celui-ci ne concerne que les personnages féminins. Ces lieux clos peuvent se situer à l'intérieur d'une maison, mais aussi en pleine nature. De plus, tous les paysages de son œuvre sont présentés comme rêvés, fictifs ou comme des réminiscences issues de l'enfance de l'écrivain. Les paysages sont de type crypté, tels des projections de signes provenant de l'inconscient du personnage féminin, où le paysage recomposé, sorti tout droit de son enfance ou de celle de l'auteur, est de l'ordre du fantasme. Les signes et symboles qui peuplent le rêve de La Porte dérobée sont tous du domaine de l'inconscient. Le paysage psychanalytique créé est ainsi un amas composite de signes et de couleurs qui révèlent un état profond de mal-être de l'héroïne Annette. Ce paysage véritablement intériorisé sert de point de rupture dans une vie qu'elle ne supporte plus. Les signes de mort (entre autres la figure 
du chien tutélaire) servent de prémonition à un échec annoncé. Le paysage met en lumière la situation d'aliénation dans laquelle se tient l'héroïne. C'est bien la notion du non-lieu qui prédomine chez Marlen Haushofer. Le roman Die Wand tout comme ses autres œuvres, se déroule dans un flou temporel, flou qui est vrai aussi pour la localisation du paysage. Si les éléments paysagers de ce roman sont identifiables et imputables à un paysage alpestre classique de type symboliste, aucun indice ne permet de le situer géographiquement dans un massif montagneux donné (absence d'indications topographiques). Si l'espace est le ferment du roman, il faut noter sa vacuité. Si l'on se réfère à l'œuvre de Norbert Reichel, Der erzählte Raum ${ }^{9}$, qui explique la mouvance et l'imprécision de la fixation du lieu, ainsi que la mise en question de sa réalité, on se rend compte de la vacuité doublée de l'irréalité des paysages du monde romanesque de Haushofer. La nature y apparaît pourtant par moments comme lieu de l'idylle (d'emblée le lieu est ainsi suspect de par la beauté des sites décrits), mais ces lieux fonctionnent aussi comme lieux de mort. Constamment la nature apparaît comme ambivalente, ayant un double fond, elle reste liée au thème de la mort (fonction de nature-mère, d'utérus et de tombe en même temps). Le paysage devient également une occasion de contemplation et de communion avec la nature et les animaux ${ }^{10}$. La symbolique de la forêt, de la montagne et de la nature montre, selon les critères de C. G. Jung, que dans toute l'œuvre le décor naturel a des traits panthéistes. De ce fait, le paysage est le lieu de la transcendance, même si presque tous les animaux qui entourent la narratrice sont de sexe masculin (sauf la vache et la chatte). Les paysages chez Marlen Haushofer véhiculent une philosophie existentialiste pessimiste et la notion du lieu clos reflète la conception du monde de cet auteur.

\section{L'écriture comme vecteur de survie psychologique}

Il est aujourd'hui couramment admis que l'écriture concourt à préciser ou à cerner l'identité d'un écrivain, mais le fait de savoir pourquoi et comment une femme écrit, reste sans réponse précise, tant les champs d'argumentation sont vastes. Si on examine dans quel domaine de la littérature les femmes ont débuté, le roman, avec ses grandes possibilités d'expression, s'impose presque autant que le genre autobiographique (journal, ouvrage de type épistolaire). Marlen Haushofer se situe à la croisée de ces deux chemins, en cryptant les éléments privés qu'elle intègre dans l'amalgame (au sens chimique du terme) de ses textes. Elle a choisi de faire évoluer ses héroïnes dans un monde du quotidien féminin (pourtant ce sont presque toutes des femmes qui ont un emploi), mais elles occupent des positions peu élevées dans la société.

Haushofer fait converger le temps et le lieu dans une suspension contemplative. En s'appuyant sur Heidegger ${ }^{11}$, on peut montrer que la suspension du temps a comme corollaire une imprécision du lieu, car la temporalité du personnage se concrétise dans sa localisation :

Das Element, das allgemein den Inhalt eines Werkes erschließt, ist der Raum. In diesem Werk sind es Orte, wo die Autorin zu Hause war: durch die Art ihrer Darstellung wirken sie jedoch nicht schonend und beruhigend, sie unterstreichen eher die Ortlosigkeit, vielleicht Heimatlo-sigkeit der Schriftstellerin, die an Mangel und Schrecken der weiblichen Figuren und an ihren Seelenzuständen gemessen werden kann. Obwohl es typische österreichische Landschaften sind, herrscht Raumlosigkeit : Sie sind kein Ort des Glücks, der Hoffnung, des sorgenfreien Lebens. Indizien des Schicksals der weiblichen Figuren befinden sich im Raum, wo sie 
unglücklich und unzufrieden leben, wo sie am Leben, ja am Zusammenleben scheitern. Die Raumlosigkeit ist hier eine Konsequenz der Raumflucht in eine übernatürliche Region, in der die Zeit aufgehoben ist. Die Zeiterfassung ist in jedem Werk Haushofers eine unpräzise, zeitgenössisch aber undatiert ${ }^{12}$.

La possibilité du monde pour une existence humaine nécessite un horizon temporel, selon Heidegger. Or, cet horizon ou cadre temporel n'existe pas dans l'œuvre de cet écrivain, les indices temporels sont flous (dans toute l'œuvre), ils sont contemporains, mais non-datés. Du fait de la non-fixation temporelle, le roman est de type presque fantastique et bénéficie également d'un no man's land temporel. Ici aussi la suspension $\mathrm{du}$ temps souligne une action hors-norme (paradoxalement celle-ci est pourtant bien réelle). Sa suspension totale est menacée par la thématique de la mort et de la remémoration ${ }^{13}$. La mise hors-temps du récit, son absence de cadrage topologique aussi, concourent à lui donner une dimension transcendantale intemporelle ${ }^{14}$. Le chiffrage des symboles de son monde littéraire et la transgression des limites qui l'accompagne montre une humanité perdue. L'atmosphère de son œuvre provoque un sentiment d'oppression (le terme allemand de Bedrückung est très juste ici) que ressent le lecteur. Mais il existe chez Haushofer une notion de transcendance et de communication avec l'au-delà du monde réel qui est un peu réconfortant. Dans un langage simple et accessible, elle illustre l'inconscient et les essais infructueux d'émancipation des femmes et en fait l'enjeu de sa création littéraire.

De la même manière que Friederike Mayröcker ou Elisabeth Reichart, Haushofer est à la recherche d'une littérature qui dirait l'indicible du monde féminin étriqué et enfermé dans des traditions figées. Comme chez ces femmes-écrivains, son œuvre est marquée par le conflit mère-fille, majeur en psychanalyse depuis les années 1960. L'écrasement, l'exclusion, le manque, sont les concepts récurrents des relations mère-fille et des relations tout court. Entre la position du père et celle de la mère, ces femmes se cherchent une voix propre, notamment par le biais de l'écriture. Si les problèmes existentiels féminins ont mobilisé les penseurs-femmes dans les années 1960 à 1970, des sociologues et non des moindres s'y sont aussi attelés (Michel Foucault, Roland Barthes, Jacques Derrida, par exemple), ce qui montre bien la complexité du champ existentiel à examiner. Le mal-être y trône en maître; il nous interroge sur l'identité de cette littérature autrichienne, dont il est une des marques, et sur la critique sociale par des intellectuels (tel par exemple Robert Menasse ${ }^{15}$ ).

17 Écrite sous le signe de la séparation et des limites, toute l'œuvre romanesque de Marlen Haushofer montre une existence de femme solitaire dans la société moderne. Les personnages ne trouvent pas leur place dans la société et se retirent dans un isolement irrévocable derrière des cloisons, dans des lieux clos. Chez Marlen Haushofer, le malêtre est individuel, il signifie la perte des sentiments et des émotions, la vie est teintée d'une profonde angoisse de mort. Sous le signe de l'enfermement et de la claustration, son œuvre est traversée par le thème de la solitude de la femme face ou à côté de son partenaire, dans un monde hostile et étriqué, régi par les lois du patriarcat. Cette autorité constitue la base de l'exclusion des femmes des décisions et des responsabilités publiques ou privées. Le silence est la seule alternative à la violence entre les sexes. Le monde extérieur à la maison est le territoire masculin, le foyer et le quotidien restant ceux de la femme. Toute son œuvre est marquée par le surgissement de l'inquiétude et $\mathrm{du}$ fantasme dans l'innocence du quotidien des héroïnes. Le rapport très fort avec les animaux est mis en regard à celui d'avec les humains. 

qu'a joué dans sa vie de femme dans une province autrichienne, l'enjeu de la création littéraire. Elle a donné à son œuvre une orientation de type transcendantal, dans une époque où le nihilisme et l'existentialisme étaient de mise. De ce fait, son œuvre se veut être une contribution au débat d'idées à partir des années 1950 (contexte postexistentialiste et structuraliste). Par le chiffrage des symboles de son monde littéraire, Marlen Haushofer se pose la question de l'existence d'une alternative pour une humanité perdue. Combat contre la folie et la mort, absence de communication entre les êtres, on retrouve dans son œuvre des thèmes classiques de la littérature autrichienne contemporaine.

\section{NOTES}

1. Romancière autrichienne, elle est née à Frauenstein (Haut-Autriche) en 1920. Après des études partielles de philologie à Vienne, elle vivra jusqu'à son décès dans la petite ville de Steyr (HauteAutriche). Mère de famille et femme d'intérieur, elle utilise les moments de répit et la nuit pour écrire. Son œuvre se compose de romans, de nouvelles et de pièces de théâtre. Elle est décédée en 1970 d'un cancer.

2. Marlen Haushofer, Die Tapetentür, Roman, Wien, Paul Zsolnay Verlag, 1957.

3. Régine Battiston-Zuliani, «La Porte dérobée ou la limite dans l'œuvre de Maren Haushofer », in Dominique Meyer-Bolzinger, Portes et fenêtres, Créliana, volume 3, printemps 2004, p. 110-119, ici p. 111.

4. La couleur jaune du chien rappelle la lumière, son ardeur et son symbole de vie - il rappelle aussi celui de la jeunesse de la narratrice. Mais on trouve aussi cette couleur dans les chambres funéraires pour assurer la survie de l'âme ainsi que chez tous les psychopompes. De par son nom, Pluto, il est lié sans équivoque au dieu des enfers. Voir aussi Jean Chevalier, Alain Gheerbrant, Dictionnaire des symboles, Robert Laffont, Paris 1982, p. 239.

5. Marlen Haushofer, Die Wand, Roman, dtv, München 1999.

6. Marlen Haushofer, Die Mansarde, Roman, Claasen, Hamburg u. Düsseldorf 1984.

7. On retrouve aussi ce fait dans l'œuvre d'I. Bachmann, voir notre analyse dans l'étude : « Malina d'Ingeborg Bachmann. Une relation triangulaire? », in Laurence Dahan-Gaida (Éd.), Logiques du Tiers, Littérature, Culture, Société, Presses Universitaires Franc-Comtoises, Annales Littéraires de l'Université de Franche-Comté, 2007, p. 181-194. Nous y avons montré que le lieu clos est un motif récurrent dans son œuvre et qu'il est associé à des « images de répression patriarcale dans des scènes primitives".

8. Régine Battiston-Zuliani, «La Porte dérobée ou la limite dans l'œuvre de Maren Haushofer », in Dominique Meyer-Bolzinger, Portes et fenêtres, op. cit., p. 117.

9. Norbert Reichel, Der erzählte Raum, Darmstadt, Wissenschaftliche Buchgesellschaft, 1987, p. 4.

10. Voir sur ce point notre étude : "Landschaftsspiegelungen in Marlen Haushofers Werk », in Régine Battiston-Zuliani (Éd.), Nature et paysages : un enjeu autrichien, Peter Lang, Berne, 2004, p. 161-175.

11. Martin Heidegger, Sein und Zeit. Niemeyer, Tübingen 1984, p. 365 et suivantes.

12. Régine Battiston-Zuliani : "Landschaftsspiegelungen in Marlen Haushofers Werk", in Régine Battiston-Zuliani (Éd.), Nature et paysages : un enjeu autrichien, op. cit., p. 162. 
13. Ibid., p. 163.

14. Voir la conclusion de notre étude, ibid., p. 174-175.

15. Cf. Robert Menasse, Das Land ohne Eigenschaften, Essay zur österreichischen Identität, Wien, Suhrkamp, 1993.

\section{RÉSUMÉS}

Écrite sous le signe de la séparation et des limites, l'œuvre romanesque de Marlen Haushofer (1920-1970) montre une existence de femme solitaire dans la société moderne. Les personnages se retirent dans un isolement derrière des cloisons ou dans des lieux clos, le mal-être est individuel, il signifie perte des sentiments et des émotions. La vie est teintée d'une profonde angoisse de mort, elle est placée sous le signe du patriarcat. Toute son œuvre est marquée par le surgissement de l'inquiétude et du fantasme dans l'innocence du quotidien. Le rapport très fort avec les animaux est mis en regard à celui d'avec les humains. Elle remet en question le principe de réalité de la vie dans un combat engagé contre les habitudes de pensée. La langue simple et accessible frappe par son réalisme. Combat contre la folie et la mort, absence de communication entre les êtres, on retrouve dans son œuvre des thèmes classiques de la littérature autrichienne contemporaine.

Das Romanwerk von Marlen Haushofer (1920-1970), das unter dem Zeichen der Trennung und der Grenzen geschrieben ist, zeigt eine einsame Frauenexistenz in der modernen Gesellschaft. Die Figuren ziehen sich in eine Isolation hinter Trennwänden oder in abgeschlossene Orte zurück, sie leiden an Schwermut, die Verlust der Gefühle und der Emotionen bedeutet. Das Leben, das von einer tiefen Todesangst gefärbt ist, ist dem Patriarchat untergeordnet. Ihr ganzes Werk kennzeichnet sich durch das Erscheinen von Angst und Wahnvorstellungen in der Alltagsunschuld. Die sehr starke Beziehung zu der Tierwelt wird der Menschenwelt gegenübergestellt. Das Werk stellt den Grundsatz der Lebenswirklichkeit durch den Kampf gegen Gedankengewohnheiten in Frage. Die einfache und zugängliche Sprache fällt durch ihren Realismus auf. Ihr Werk weist klassische Themen der zeitgenössischen österreichischen Literatur wie der Kampf gegen Wahnsinn und Tod, die Kommunikationslosigkeit zwischen den Wesen auf.

Written under the sign of separation and limits, the novels of Marlen Haushofer (1920-1970) show the solitary existence of women in modern society. The characters withdraw in isolation behind partitions or in enclosed places, discomfort is individual, it means loss of feelings and emotions. Life is tinged with a deep anguish of death; it is placed under the sign of the patriarchate. All of Haushofer's work is marked by the sudden appearance of anxiety and phantasm in the innocence of daily life. The very strong bond with animals is seen though the eyes of human relations. She calls into question the principle of the reality of life in a struggle against usual thinking. Her simple and accessible language strikes by its realism. Her work contains the traditional topics of contemporary Austrian literature, such as : the fight against madness and death, and lack of communication between human beings. 
AUTEUR

RÉGINE BATTISTON

Université de Haute-Alsace 